\title{
Baryons in 2+1 flavour domain wall QCD
}

\section{D.J. Antonio, K.C. Bowler, P.A. Boyle, M.A. Clark, B. Joó, A.D. Kennedy, R.D. Kenway, C.M. Maynard*, R.J. Tweedie.}

School of Physics, University of Edinburgh, Edinburgh, EH9 3JZ, UK.

E-mail:

\begin{tabular}{|l|}
\hline s0459477@sms.ed.ac.uk \\
\hline kcb@ph.ed.ac.uk. \\
\hline paboyle@ph.ed.ac.uk \\
\hline mike@ph.ed.ac.uk \\
\hline bj@ph.ed.ac.uk \\
\hline adk@ph.ed.ac.uk \\
\hline r.d.kenway@ed.ac.uk \\
\hline cmaynard@ph.ed.ac.uk \\
\hline rjt@ph.ed.ac.uk \\
\hline
\end{tabular}

\section{A. Yamaguchi}

Department of Physics and Astronomy, University of Glasgow, Glasgow, G12 8QQ, UK.

E-mail:

a.yamaguchi@physics.gla.ac.uk

\section{RBC and UKQCD Collaborations}

We present results for some of the light baryon masses and their excited states in $2+1$ flavour domain wall QCD. We considered several lattice spacings, with the DBW2 and Iwasaki gauge actions and different sea quark masses on a volume of $16^{3} \times 32$ and a fifth dimension of size 8 . All data were generated on the QCDOC machines. Despite large residual massses and a limited number of sea quark mass values with which to perform chiral extrapolations, our results are in reasonable agreement with experiment and scale within errors. Finite size effects on most ensembles appear to be small.

XXIIIrd International Symposium on Lattice Field Theory

Trinity College, Dublin, Ireland

25-30 July 2005

\footnotetext{
*Speaker.
} 


\section{Introduction}

The calculation of many quantities from lattice QCD with quenched, or heavy dynamical, quarks has produced results which, although in qualitative agreement with experiment, have uncontrolled sources of error. To progress beyond this, calculations with light dynamical quarks are necessary. Using fermions which satisfy the Ginsparg-Wilson (GW) relation is advantageous, as the theory is well defined within broad parameter ranges. It has lattice versions of the symmetries of continuum QCD, and the correct flavour content. Mixing of operators with different chirality is suppressed, renormalisation is simplified, and a continuum-like chiral perturbation theory can be used for the extrapolation of quantities to the chiral limit. Domain wall fermions (DWF) satisfy these requirements. The QCDOC machine and the RHMC algorithm have allowed for the first time calculations using DWF with $2+1$ flavours of dynamical quarks.

Baryon physics is an area rich in phenomenology. Unanswered questions as to the nature of some of the excited states, the decay of the proton predicted by some Grand Unified and super symmetric models, and the determination of matrix elements related to the structure functions and the neutron electric dipole moment can, in principle, be determined by lattice QCD calculations. These calculations are very computationally challenging, as they need both light sea quarks and large volumes, as well as fermions with the correct symmetry properties. In this work we report on a study of the lowest lying states in the baryon spectrum, $\left\{N, \Delta, \Omega, N^{\star}\right\}$, on ensembles of configurations produced by QCDOC. It is important for any calculation claiming to be "full QCD" to be able to reproduce this spectrum. These initial ensembles were produced primarily for a search of parameter space to guide larger production runs. As such, they have a small volume, too small certainly for excited states such as the $N^{\star}$, but this can be used, eventually in combination with the productions runs on larger volumes to try and estimate the size of the finite volume effects on the remaining spectrum.

The DWF action is given in [1] with the Pauli-Villars field in [2] for the dynamical simulation. The gauge fields were generated with renormalisation group (RG) improved actions, as follows:

$$
S_{G}=-\frac{\beta}{3}\left[(1-8) c_{1} \sum_{x, \mu v} P(x)_{\mu v}+c_{1} \sum_{x, \mu \neq v} R(x)_{\mu v}\right]
$$

with either $c_{1}=-1.4069$ for the DBW2 action [3] or $c_{1}=-0.331$ for the Iwasaki action [4]. It has been noted in the quenched DWF calculations [5] that these actions reduce the chiral symmetry breaking resulting from the finite fifth dimension. The mechanism and size of chiral symmetry breaking on these ensembles is studied in detail in [6].

In the DWF formalism, the 4D quark fields are constructed from left and right projections of the 5D fermion fields on the boundaries. With a finite fifth dimension, there is still an overlap between these left- and right-handed fields, which manifests itself as an additive mass renormalisation, known as the residual mass. This can be determined directly from the axial Ward-Takahashi identity

$$
\begin{aligned}
\partial_{\mu} A_{\mu}(x) & =2 \operatorname{am}_{f} P(x)+2 J_{5}(x) \\
& \approx 2\left(a m_{f}+a m_{\mathrm{res}}\right) P(x)
\end{aligned}
$$




\begin{tabular}{|c|c|c|c|c|c|c|c|}
\hline$\beta$ & $a m_{l} / a m_{s}$ & $r_{0} / a$ & $a^{-1}(\mathrm{GeV})$ & $L(\mathrm{fm})$ & $\operatorname{Lm}_{P}$ & $m_{P} / m_{V}$ & \#traj \\
\hline 0.72 & $0.04 / 0.04$ & \multirow{2}{*}{$4.3(1)$} & \multirow{2}{*}{$1.7(1)$} & \multirow{2}{*}{$1.9(1)$} & $7.7(1)$ & $0.692(5)$ & 3400 \\
\hline 0.72 & $0.02 / 0.04$ & & & & $6.0(1)$ & $0.589(3)$ & 6000 \\
\hline 0.764 & $0.04 / 0.04$ & \multirow{2}{*}{$5.1(2)$} & \multirow{2}{*}{$2.0(1)$} & \multirow{2}{*}{$1.6(1)$} & $6.7(1)$ & $0.699(4)$ & 5750 \\
\hline 0.764 & $0.02 / 0.04$ & & & & $5.1(1)$ & $0.619(4)$ & 3000 \\
\hline 2.13 & $0.04 / 0.04$ & \multirow{2}{*}{$4.6(2)$} & \multirow{2}{*}{$1.8(1)$} & \multirow{2}{*}{$1.8(1)$} & $7.5(1)$ & $0.700(8)$ & 3600 \\
\hline 2.13 & $0.02 / 0.04$ & & & & $5.8(1)$ & $0.615(5)$ & 3600 \\
\hline 2.2 & $0.04 / 0.04$ & \multirow{2}{*}{$5.3(1)$} & \multirow{2}{*}{$2.1(1)$} & \multirow{2}{*}{$1.5(1)$} & $6.8(1)$ & $0.726(2)$ & 4500 \\
\hline 2.2 & $0.02 / 0.04$ & & & & $5.1(1)$ & $0.667(8)$ & 3200 \\
\hline
\end{tabular}

Table 1: Properties of the ensembles used in this study. The value of $r_{0} / a$ was determined in [9]. The lattice spacing, and thus the volume are set by choosing $r_{0}=0.5 \mathrm{fm}$. Trajectories are of length 0.5

where $J_{5}(x)$ is the point-split current constructed from fields at the mid-point of the fifth dimension and $P(x)$ the pseudoscalar density.

\section{Details of the calculation}

The ensembles were all generated on QCDOC machines, using the exact RHMC algorithm [7] 8], with a volume of $16^{3} \times 32$ and a fifth dimension of size $L_{S}=8$ and are detailed in Table 1. The integrated autocorrelation times are estimated to be $\mathscr{O}(50)$ for mesonic correlators. To maximise statistics, the correlation functions were measured every 5 trajectories, and then binned so that the separation between independent measurements is 100 trajectories. The correlators were computed from up to four sources on different time-planes with as many as three different smeared sources.

The masses of the pseudoscalar and vector mesons were determined by simultaneous fits to the local and smeared correlators with two exponentials, the ground state and the excited state. Similarly $m_{\text {res }}$ was determined by a simultaneous fit of a plateau to a ratio of correlators, with both smeared and local sources.

The standard baryon interpolating operator is given by

$$
\Omega(x)=\varepsilon_{i j k}\left[\psi_{i}(x) C \Gamma \psi_{j}(x)\right] \psi_{k}(x)
$$

For the $I=\left\{\frac{1}{2}, \frac{3}{2}\right\}$ baryons, $\Gamma=\left\{\gamma_{5}, \gamma_{5} \gamma_{k}\right\}$. Another operator, which projects onto the negative parity $I=\frac{1}{2}$ state, with $\Gamma=1$ was also used. For baryon correlators in a finite box with periodic boundary conditions, the backward propagating state is the negative parity partner, that is

$$
C_{B}(t)=A_{+} e^{-m_{+} t}+A_{-} e^{-m_{-}(T-t)}
$$

For the $I=\frac{1}{2}$ baryon, the masses of the positive and negative parity states were determined by a simultaneous fit to equation (2.2) using the standard operator, and a single exponential to the negative parity correlator. This is shown in Figure [1(a). Typically this was computed for the smeared correlator only, as the local correlator had a poor signal.

The mass of the $I=\frac{3}{2}$ baryon was determined from a single exponential fit to the smeared correlator, as the signal for the excited, negative parity partner was poor, as might be expected on relatively small numbers of configurations. 
(a)

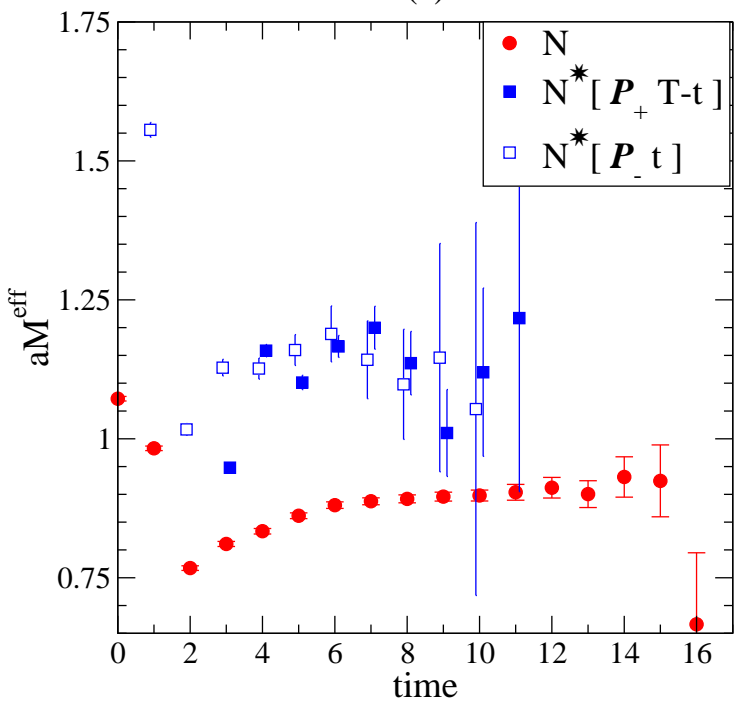

(b)

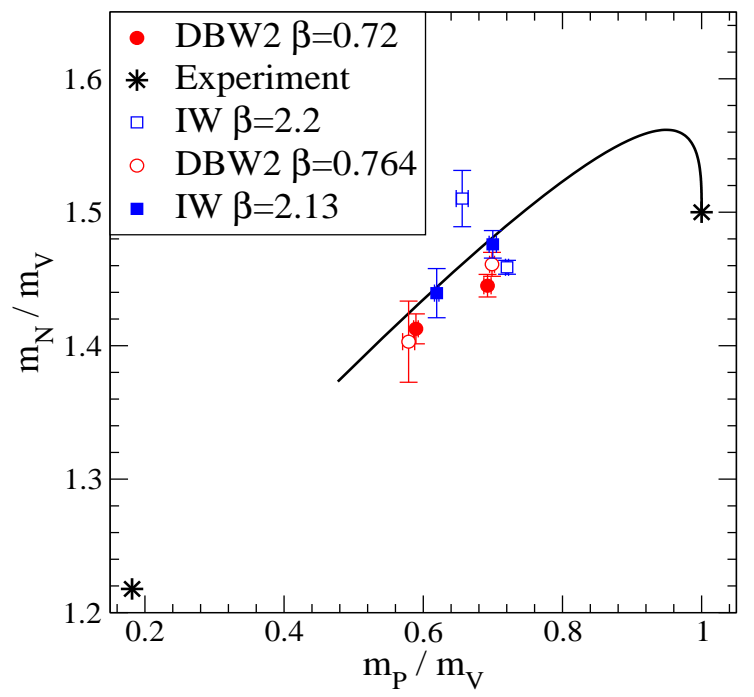

Figure 1: (a) The effective mass of $I=\frac{1}{2}$ baryon correlator on the DBW2 $\beta=0.72, \mathrm{am}_{\mathrm{l}} / \mathrm{am}_{\mathrm{s}}=0.02 / 0.04$ ensemble. The red symbol shows the data for the positive parity state, the closed blue squares are the timereversed backward propagating negative parity state data and the open blue squares are the negative parity data from the second correlator. (b) The Edinburgh plot for the different ensembles.

\section{Preliminary Results}

Shown in Figure 1(b) is the Edinburgh plot. It is reassuring that, even at relatively coarse lattice spacing, with a small fifth dimension and consequently moderate chiral symmetry breaking, the data follows the phenomenological curve very well. The only exception is the lightest Iwasaki $\beta=2.2$ datum. Naively, one might expect this to be a finite size effect, especially, examining Table 1, given that the lattice spacing and thus the box size are rather small. However, the value of $L m_{P}$ is not significantly smaller than the other data sets and, critically, $L m_{P}>4$, suggesting that the box is big enough as measured by the pseudoscalar meson.

For this particular ensemble the signal for the vector meson mass is not good. In particular, the effective mass plot has a poor plateau, and a stable fit can only be achieved for the lowest region. The net effect is for a rather low vector meson mass. It is probable that this is due simply to low statistics. A low estimate of the vector mass would cause this datum in the Edinburgh plot to be shifted up and to the right, which could be mistaken for a finite volume effect increasing the mass of the nucleon.

The quark mass was defined as

$$
a m_{q}=a m_{f}+a m_{\mathrm{res}}\left(m_{f}\right)
$$

with the chiral limit at $a m_{q}=0$. With two sea quark masses, only a crude chiral extrapolation could be attempted, i.e. drawing a straight line through the two data points. The strange quark mass, (for the $\Omega$ baryon) was set from the kaon mass.

Shown in Figure $\mathrm{Z}$ is the dependence of the baryon spectrum, in dimensionless units, on the lattice spacing. A continuum extrapolation cannot be attempted with these ensembles. However, 


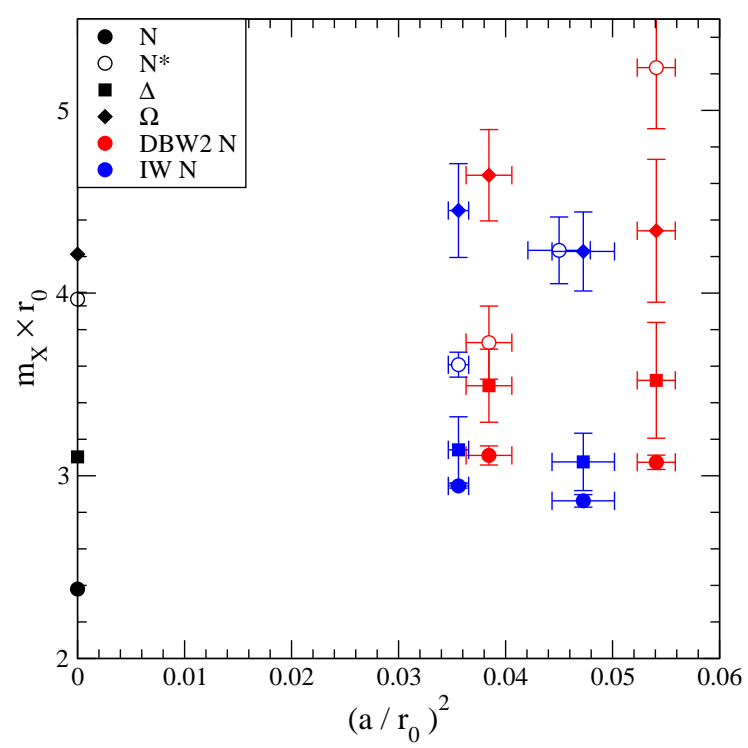

Figure 2: Scaling of the baryon spectrum with lattice spacing squared. The symbols denote the following: Closed circles $N$, open circles $N^{\star}$, squares $\Delta$, diamonds $\Omega$. Black symbols denote experiment, red DBW2 ensembles, blue Iwasaki ensembles. The value of $r_{0}=0.5 \mathrm{fm}$ was chosen, to give an indication of the experimental spectrum in these units. The $N^{\star}$ measured on the $\beta=2.13$ ensemble (the furthest right open blue circle) has been offset to the left for clarity.

the data for the ground states, $\{N, \Delta, \Omega\}$, shows reasonable scaling, albeit with large errors. These large uncertainties are due to the crude nature of the chiral extrapolation. The determination of $r_{0}$ is well defined for each ensemble, and thus it is a good quantity to use to examine scaling behaviour. As the value of $r_{0}$ in the continuum is unknown, rather than setting the absolute scale with $r_{0}$, a better strategy is to predict dimensionless ratios of physical quantities in the continuum, while using $r_{0}$ to just examine the scaling behaviour.

The negative parity partner of the nucleon, the $N^{\star}$ is expected to become degenerate with the nucleon in a small enough box. This effect can be clearly seen from Figure ㄴ the $N^{\star}$ mass drops dramatically as the volume is reduced. This suggests that finite size effects may also be beginning to affect the ground states for the ensembles at finest lattice spacing. These finite size effects would tend increase the mass of the ground states. The slight upward tendancy in the scaling plot as the lattice spacing decreases is consistent with the finite size effects spoiling otherwise very good scaling, or a small scaling violation for the nucleon mass.

\section{Conclusions}

We have determined the spectrum of the lowest lying baryon states for several ensembles with two different gauge actions. The QCDOC machines and the RHMC algorithm have made $2+1$ flavours of DWF ensembles possible for the first time. With limited statistics, and only two different sea quark masses, we qualitatively reproduce the experimental spectrum. There is limited evidence that finite size effects may be influencing the ground state baryons on the smaller volumes. 
Both the Edinburgh plot, and the scaling analysis suggest that a programme of baryon physics on larger volumes and at lighter quark mass will yield very interesting results.

\section{Acknowledgements}

We thank Saul Cohen, Sam Li and Meifeng Lin for help generating the datasets used in this work. We thank Dong Chen, Norman Christ, Saul Cohen, Calin Cristian, Zhihua Dong, Alan Gara, Andrew Jackson, Chulwoo Jung, Changhoan Kim, Ludmila Levkova, Xiaodong Liao, Guofeng Liu, Robert Mawhinney, Shigemi Ohta, Konstantin Petrov and Tilo Wettig for developing with us the QCDOC machine and its software. This development and the resulting computer equipment used in this calculation were funded by the U.S. DOE grant DE-FG02-92ER40699, PPARC JIF grant PPA/J/S/1998/00756 and by RIKEN. This work was supported by PPARC grant $\mathrm{PPA} / \mathrm{G} / \mathrm{O} / 2002 / 00465$.

\section{References}

[1] V. Furman and Y. Shamir, Axial symmetries in lattice qcd with kaplan fermions, Nucl. Phys. B439 (1995) 54-78 [hep-lat/9405004].

[2] P. M. Vranas, Chiral symmetry restoration in the schwinger model with domain wall fermions, Phys. Rev. D57 (1998) 1415-1432 [hep-lat/9705023].

[3] QCD-TARO Collaboration, P. de Forcrand et. al., Renormalization group flow of su(3) lattice gauge theory: Numerical studies in a two coupling space, Nucl. Phys. B577 (2000) 263-278 hep-lat/9911033.

[4] Y. Iwasaki and T. Yoshie, Renormalization group improved action for su(3) lattice gauge theory and the string tension, Phys. Lett. B143 (1984) 449.

[5] T. Blum et. al., Quenched lattice qcd with domain wall fermions and the chiral limit, Phys. Rev. D69 (2004) 074502 [hep-lat/0007038].

[6] P. Boyle. Localisation and chiral symmetry in 2+1 flavour domain wall $Q C D$, in proceedings of XXIIIrd International Symposium on Lattice Field Theory, PoS(LAT2005)141.

[7] A. D. Kennedy, I. Horvath and S. Sint, A new exact method for dynamical fermion computations with non-local actions, Nucl. Phys. Proc. Suppl. 73 (1999) 834-836 [hep-lat/9809092].

[8] M. Clark. Algorithm Shootout: R versus RHMC, in proceedings of XXIIIrd International Symposium on Lattice Field Theory, PoS(LAT2005)115.

[9] K. Hashimoto, T. Izubuchi and J. Noaki. The static quark potential in 2+1 flavour Domain Wall QCD from QCDOC, in proceedings of XXIIIrd International Symposium on Lattice Field Theory, PoS(LAT2005)093. 